TROPICAL MEDICINE SERIES

\title{
Trichomoniasis: clinical manifestations, diagnosis and management
}

\author{
H Swygard, A C Seña, M M Hobbs, M S Cohen
}

Sex Transm Infect 2004;80:91-95. doi: 10.1136/sti.2003.005124

Trichomonas vaginalis was originally considered a commensal organism until the 1950s when the understanding of its role as a sexually transmitted infection (STI) began to evolve. Trichomoniasis has been associated with vaginitis, cervicitis, urethritis, pelvic inflammatory disease (PID), and adverse birth outcomes. Infection with $T$ vaginalis could have an important role in transmission and acquisition of HIV. T vaginalis is site specific for the genitourinary tract and has been isolated from virtually all genitourinary structures. Asymptomatic disease is common in both men and women, thus screening for disease is important. Various sociodemographic factors have been correlated with presence of $T$ vaginalis, and may be used to predict infection. Diagnosis is usually made from wet mount microscopy and direct visualisation, which are insensitive. DNA amplification techniques perform with good sensitivity, but are not yet approved for diagnostic purposes. In areas where diagnostic methods are limited, management of trichomoniasis is usually as part of a clinical syndrome; vaginal discharge for women and urethral discharge for men. A single dose of metronidazole is effective in the majority of cases. Outside of the United States, other nitroimidazoles may be used and are as effective as metronidazole. Metronidazole resistance is an emerging problem, but its clinical importance is not yet clear. Concomitant treatment of sexual partners is recommended.

Series editor: David Lewis

See end of article for authors' affiliations

Correspondence to: Heidi Swygard, CB \# 7030, 130 Mason Farm Road, The University of North Carolina at Chapel Hill, Chapel Hill, NC 27599-7030, USA: Heidi_Swygard@ med.unc.edu

Accepted for publication 1 September 2003
T richomoniasis is a sexually transmitted disease (STI) with important public health ramifications; it has been associated with vaginitis, cervicitis, urethritis, and pelvic inflammatory disease (PID). Trichomoniasis also impacts upon birth outcomes and is a co-factor in human immunodeficiency virus (HIV) transmission and acquisition. ${ }^{1}$

Approximately 180 million women worldwide may be infected with $T$ vaginalis. Prevalence estimates vary between populations studied, but range from $5-74 \%$ in women and $5-29 \%$ in men, with the highest rates reported in either sex from STI clinics and in other high risk populations. $^{2-5}$ In men, the prevalence is less well described because many men are asymptomatic and may not seek evaluation. Furthermore, the diagnosis is less commonly sought in men. ${ }^{6}$ In either sex, concomitant infection with other sexually transmitted organisms occurs commonly. ${ }^{78}$ Because of the public health importance of this common STI, we review here the clinical aspects of trichomoniasis.

\section{METHODS}

Using combinations of the keyword "trichomoniasis" with "epidemiology," "diagnosis," "manifestations," and "therapy," a Medline search was performed. The search was limited to English language articles published in the previous 10 years. Editorials, letters to the editor, case reports, and case series were excluded. The resulting titles were searched for and limited to "trichomoniasis" or "Trichomonas vaginalis." Resulting abstracts were reviewed and articles were excluded if the focus was not clinically relevant. Articles representing a developing body of literature were limited to the most recent publication date.

\section{RESULTS}

A total of 396 articles were retrieved through the Medline search. Subsequent review by the authors resulted in 195 articles with "trichomoniasis" or "T vaginalis" in the title. Abstracts $(\mathrm{n}=94)$ were reviewed; 52 of these were selected and reviewed here.

\section{Epidemiology}

Among US women, race has been identified in several studies as predictive of infection with $T$ vaginalis." This association is probably multifactorial, including access to care, personal health practices, and socioeconomic factors. ${ }^{1}$ Older age, histories of previous STI, prostitution, pregnancy, and drug use have also been associated with trichomoniasis. ${ }^{10}{ }^{11}$ The association with older age is in contrast with the association between age and other STIs, especially chlamydia. Reproductive hormone levels may be partly responsible for higher prevalence of trichomoniasis in older women. ${ }^{12}$ Among African women, vaginitis including trichomonal vaginitis was significantly associated with sexual debut at less than 20 years, a partner who travelled frequently, history of STI, and presence of abnormal discharge. ${ }^{13}$ Concomitant STI diagnosis is common (15-28\% chlamydial co-infection and 10\% gonorrhoeal co-infection rates) in women with trichomoniasis, indicating the importance of screening for other STIs. ${ }^{14}$

Among US men attending STI clinics, the combination of age $\geqslant 30$ years, discharge, and diagnosis of non-gonococcal urethritis (NGU) was predictive of infection with $T$ vaginalis, 
although this finding was not supported in a separate study. ${ }^{16}{ }^{17}$ Sexual exposure to a woman with trichomoniasis and history of trichomoniasis were also associated with trichomoniasis in multivariate analyses. ${ }^{16}$ As in women, concomitant infection with other urethral pathogens is common in trichomoniasis. Mixed infections with $T$ vaginalis and Neisseria gonorrhoeae and/or Chlamydia trachomatis occurred in seven of 980 rural Tanzanian men, while $61.5 \%$ of 91 men with trichomoniasis in west Africa also had a gonococcal infection. ${ }^{818} 19$

Finally, trichomoniasis facilitates spread of the HIV epidemic. One theoretical calculation concludes that if infection with $T$ vaginalis increased the risk of HIV transmission by $90 \%$ in a population with $25 \%$ prevalence of trichomoniasis, approximately $20 \%$ of cases of HIV would be attributable to trichomoniasis. ${ }^{1}$ This relation could explain some of the differences in rates of HIV infection in different regions of Africa where rates of trichomoniasis and HIV seem to be directly proportional. ${ }^{20}$

\section{Clinical presentation \\ Women}

$T$ vaginalis is site specific for the genitourinary tract and has been isolated from virtually all genitourinary structures. However, many women diagnosed with trichomoniasis are asymptomatic; in Zimbabwe $75 \%$ of women denied symptoms on direct questioning and $16 \%$ of these women had trichomoniasis. ${ }^{13}$ When symptoms arise, the most common presenting complaint among women diagnosed with $T$ vaginalis is vaginal discharge, seen in more than $50 \%$ of cases, followed by pruritus or dysuria. One study of 200 Nigerian women demonstrated $74 \%$ with vaginal discharge were infected with $T$ vaginalis. ${ }^{2}$ On speculum examination, the vaginal discharge may be of any colour or characteristic, and although a frothy green discharge has classically been associated with trichomoniasis, this was not observed in any of 149 Nigerian women with discharge caused by trichomoniasis. ${ }^{2}$ The discharge may be malodorous with $\mathrm{pH}>4.5{ }^{7}$ Trichomoniasis has been associated with bacterial vaginosis in other studies. ${ }^{21}{ }^{22}$ Clue cells were found to be an independent risk factor for trichomoniasis among 249 South African women attending family planning clinics. ${ }^{23}$ However, these characteristics are neither sensitive nor specific. The clinician's ability to predict vaginal infection with $T$ vaginalis based solely on physical examination was shown to have a positive predictive value of $47 \%$ among commercial sex workers in Cameroon. ${ }^{24}$

Cervical pathology can be commonly seen with trichomoniasis. Colpitis macularis, or "strawberry cervix," (fig 1) results from microscopic, punctate haemorrhages of the cervix. $T$ vaginalis is significantly associated with the clinical

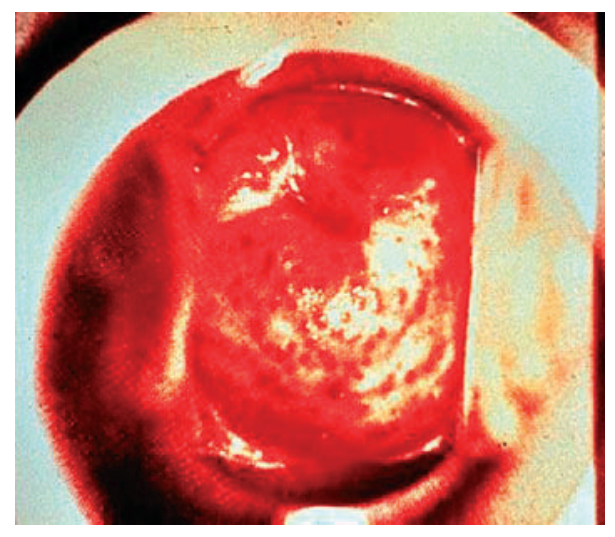

Figure 1 Colpitis macularis, or "strawberry cervix." (Courtesy, CDC.) finding of colpitis macularis. Cervical mucopurulence, erythema, and friability have been observed. ${ }^{73} T$ vaginalis may be associated with cervical cancer; however, confounding factors, such as co-infection with HPV, have not been well controlled for in some of these studies. ${ }^{25}$ Among HIV infected women, $T$ vaginalis infection was associated with PID. ${ }^{21}$

Trichomonal infections are neither restricted to nor exclusive for the vagina, and urinary tract infection may be common. Dysuria was observed in $29 \%$ of South African women diagnosed with $T$ vaginalis infection. ${ }^{23}$ Thus, consideration should be given to the presence of trichomoniasis for women with dysuria as a sole complaint.

Finally, there is some suggestion that women may harbour T vaginalis for 3-5 years. ${ }^{26}$ The reasons for this are unclear, but may include imperfect screening tools, genitourinary anatomy, or type of infecting strain.

\section{Men}

Less is known about $T$ vaginalis infection in men. Current estimates of asymptomatic infection in men vary. In one US study, 23 of 50 patients with trichomoniasis were asymptomatic. ${ }^{27}$ Among 23 east African men with trichomoniasis, 19 were asymptomatic. ${ }^{28}$ Finally, among 100 men presenting for non-genitourinary complaints in the Central African Republic, seven were diagnosed with trichomoniasis. ${ }^{8}$ When symptoms of trichomoniasis become manifest, discharge may be less profuse and purulent than that seen with gonococcal infection. ${ }^{27}$ Men may also complain of dysuria. Of 103 Tanzanian men with trichomoniasis, dysuria occurred in $42 \%$; only five men complained of urethral discharge. ${ }^{19}$ Finally, trichomoniasis was diagnosed in 66 of 410 men from the Central African Republic with urethral discharge. ${ }^{8}$ In men, urethral discharge and dysuria are the most common symptoms; however, some men diagnosed with trichomoniasis are asymptomatic. ${ }^{29}$ Many men clear $T$ vaginalis from the genitourinary tract within 2 weeks. ${ }^{30}$ Involvement of other male genitourinary structures has been described, but will not be reviewed here.

\section{Diagnosis}

\section{Specimens}

Suitable clinical specimens include urine, vaginal fluid, semen, and endocervical smear. Because of discordantly infected anatomical sites among men, testing from multiple sites is advisable. ${ }^{31}$ Vaginal specimens for culture may be either self collected with a swab, or collected by the clinician with a swab or plastic loop. ${ }^{32}$ Self collected vaginal specimens, which may have greater acceptability with patients, perform similarly to clinician acquired specimens for the diagnosis of trichomoniasis. $^{33}$

\section{Direct visualisation}

These methods depend on identification of the organism on microscopic examination of clinical specimens. Wet mount preparation and culture rely on visual detection of sufficient numbers of viable organisms with characteristic motility. Wet mount preparation has been the most commonly used method for diagnosis of trichomoniasis in women. Vaginal secretions are obtained from the lateral walls and fornices using a swab or plastic loop; in men, any urethral discharge, prostate secretions, or urethral scrapings may be used. The secretions are then suspended in $0.85 \%$ normal saline and examined under the microscope. Because the organism will quickly lose its characteristic tumbling motility, the specimen should be examined as quickly as possible after it has been obtained..$^{34}$ Although this method has imperfect sensitivity, it is both inexpensive and rapidly performed..$^{35}$

Culture has long been considered the gold standard for diagnosing $T$ vaginalis infection. Several different media are available for culture, including self contained systems. Of 
these media, Diamond's modified has been shown to be superior although the self contained system has been shown to have equivalent performance. ${ }^{36}$ Culture is more sensitive than wet mount preparation, because of the lower minimum concentration of organisms required for a positive result. ${ }^{36}$ The drawbacks to culture include greater cost and prolonged time to diagnosis (3-7 days). Screening with culture, although far more sensitive than wet mount preparation, may not be feasible; however, delayed inoculation after a negative wet mount preparation could increase detection in high prevalence populations without substantially increasing cost. $^{37}$

As a screening tool for trichomoniasis, Papanicolaou (Pap) smear performs with low sensitivity $(61 \%) .{ }^{38}$ In one study, endocervical smears were less sensitive than ectocervical smears, leading the authors to suggest that the ectocervical smear may have greater utility for detecting $T$ vaginalis on Pap smear. ${ }^{39}$ Depending on the prevalence of $T$ vaginalis in a population, almost one third of women with Pap smears positive for trichomoniasis receive unnecessary therapy. ${ }^{40}$ In a meta-analysis of the performance of Pap smear compared to wet mount preparation for the diagnosis of vaginal trichomoniasis, a three tiered approach for the interpretation of a positive Pap smear was proposed. ${ }^{41}$ In high prevalence populations $(>20 \%)$, a positive Pap smear had a positive predictive value (PPV) of $83 \%$, whereas in lower prevalence populations the PPV decreased. Confirmatory culture for $T$ vaginalis has been recommended for suspicious Pap smears in low prevalence populations. ${ }^{41}$

Other staining and molecular probe methods have been employed with direct clinical specimens..$^{42} 43$ While rapidly performed and having sensitivity similar to that of wet mount preparation, they require access to specialised equipment, skilled laboratory personnel, and sufficient financial resources.

\section{Nucleic acid amplification}

Because of the insensitivity of wet mount preparation and the problems with culture methods, nucleic acid amplification methods, such as polymerase chain reaction (PCR), have become increasingly attractive for diagnosing infection with $T$ vaginalis. PCR has the advantage of requiring only DNA, from either viable or non-viable organisms, and in concentrations as low as one organism per PCR reaction. ${ }^{44}$

PCR has been performed on several different types of clinical specimens, most notably vaginal fluid and urine. Self administered vaginal swabs have been used for PCR assays, and performed at least as well as wet mount preparation, although specificity is less than that of wet mount or culture. $^{45}$ Urine based PCR detection of $T$ vaginalis has performed with sensitivity and specificity of $64-90.8 \%$ and $93.4-100 \%$ in US women and $92.7 \%$ and $88.6 \%$ in African men, respectively. ${ }^{46}{ }^{47}$ This technology is not yet commercially available.

\section{Syndromic management}

Screening for trichomoniasis may be problematic in resource challenged settings. In such settings, the World Health Organization (WHO) has promoted syndromic approaches for managing STIs. However, using mathematical modelling, Bowden et al demonstrated that syndromic management had minimal effect on endemic prevalence, and that screening was the most efficient method of control. ${ }^{26}$ In general, syndromic management has been shown to work well for genital ulcer disease for either sex, and urethral discharge for men. The urethral discharge algorithm does not consider trichomoniasis until after the patient has been treated for gonorrhoea and chlamydia. Based on the prevalence of trichomoniasis as a cause of urethritis, some experts have suggested the addition of metronidazole or another nitroimidazole to the initial therapy in the syndromic management algorithm for urethral discharge. ${ }^{19}$ Syndromic management of vaginal discharge performs poorly for women because cervical discharge is a confounding factor. In a study of 249 South African women attending antenatal clinics, only one in three women treated for trichomoniasis based on a risk score would actually have been correctly diagnosed with trichomoniasis based on wet mount microscopy; overtreatment using syndromic management compared to microbiological diagnosis of trichomoniasis was observed in another study. ${ }^{23} 48$

\section{Treatment}

Definitive therapy for trichomoniasis became possible with the introduction of nitroimidazoles (metronidazole, tinidazole, ornidazole, carnidazole, and nimorazole). In a metaanalysis of nitroimidazoles where the majority of studies used tinidazole or metronidazole for treatment of trichomoniasis, in short or prolonged courses of oral therapy, parasitological cure resulted in $90 \%$ of cases. ${ }^{49}$ Topical metronidazole is not effective for therapy for trichomoniasis. ${ }^{50}$ The Centers for Disease Control and Prevention (CDC) has estimated that 5\% of clinical isolates of $T$ vaginalis exhibited some degree of metronidazole resistance. ${ }^{51}$ Metronidazole resistance may predict tinidazole resistance; however, it is unclear if this situation is true for the entire class of nitroimidazoles. ${ }^{52}$ The CDC has proposed an escalated therapeutic regimen for management of infection with resistant strains (fig 2). Furazolidone, a nitrofuran, was shown to be effective in vitro against both laboratory and clinical specimens with high level metronidazole resistance, and topical paromomycin

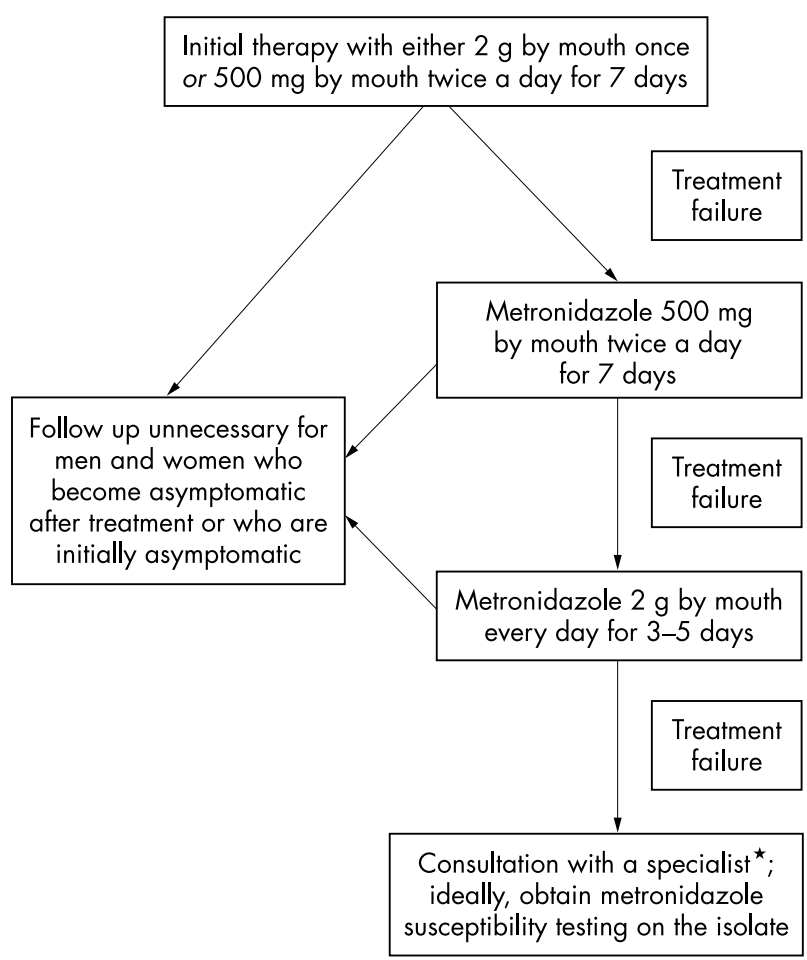

Figure 2 Guidelines for treating metronidazole resistant trichomoniasis. 
was effective in seven of 12 patients infected with resistant strains; however, paromomycin had adverse dermatological effects.5. ${ }^{53}$ Alternatives for treatment of highly resistant trichomoniasis exist but have not been subjected to the rigours of clinical trials.

Side effects of nitroimidazoles include a bitter metallic taste, nausea, and vomiting, a disulfiram-like reaction with ingestion of alcohol and some central nervous system effects. Despite the low doses required for treatment of trichomoniasis, side effects occur in more than half of patients, especially with the metronidazole $2 \mathrm{~g}$ dose. ${ }^{49}$

\section{Other management issues}

Treatment relapse usually represents re-infection by an untreated partner in the majority of cases, although newly recognised chronic or drug-resistant infections are possible. ${ }^{9}$ Re-infection is common, occurring in $36 \%$ of subjects in one study. ${ }^{11}$ Recommendations for concomitant treatment of the male partner have been made since the 1960s and are now the standard of care. ${ }^{54}$

Risk factors for re-infection with $T$ vaginalis in HIV infected women have been evaluated. In a study of 411 HIV positive women, 36\% were re-infected at least once with $T$ vaginalis over a mean follow up of 3.5 years. Predictors of re-infection or recurrent infection in these women were age $\geqslant 22$ years and history of previous STI in multivariate analysis. ${ }^{11}$

Some pregnant women with trichomoniasis may present with premature rupture of membranes, preterm labour. ${ }^{55}$ However, a randomised placebo controlled trial of 615 asymptomatic pregnant women with trichomoniasis did not demonstrate a difference in rates of preterm birth between the treatment and placebo groups. ${ }^{56}$ Although the reasons for this finding are unclear, the authors speculated that inflammatory responses to dying organisms or release of a pathogenic virus might be responsible. Because of the possible association with adverse birth outcomes, some authorities recommend treating trichomoniasis in pregnant women. ${ }^{54}$ The use of metronidazole in pregnancy has been controversial because of its potential mutagenic and carcinogenic potential. Increased risks of birth defects or teratogenicity have not documented. ${ }^{57}$ For clinicians concerned about the possibility of effects on the fetus, intravaginal therapy with metronidazole or clotrimazole could be considered as alternatives; however, studies of these therapies have demonstrated low parasitological cure rates. ${ }^{50} 58$

\section{SUMMARY}

$T$ vaginalis infection is highly prevalent in sexually active populations. Trichomoniasis is clearly associated with significant public health problems, including HIV transmission. Disease presentation is variable and diagnosis can be difficult with the most commonly used methods. Certain sociodemographic and behavioural risk factors may assist in predicting infection. Treatment of trichomoniasis with one dose of metronidazole is highly effective, and should be delivered in conjunction with concomitant treatment of sexual partners.

A majority of women and a significant proportion of men with trichomoniasis are without any symptoms; these patients would thus escape detection and treatment under syndromic management recommendations. Control programmes must work towards increased screening in order to have any effect on the burden of STIs.

\section{CONTRIBUTORS}

HS and ACS, identification of articles for review; HS and ACS, analysis and interpretation; HS, drafting of the article; ACS, MMH, MSC, critical revision for content; ACS, MMH, MSC, final approval; HS, collection and assembly of articles.

\section{Authors' affiliations}

H Swygard, A C Seña, M M Hobbs, M S Cohen, The University of North Carolina at Chapel Hill, Chapel Hill, NC, USA

\section{REFERENCES}

1 Sorvillo F, Smith L, Kerndt $P$, et al. Trichomonas vaginalis, HIV and AfricanAmericans. Emerg Infect Dis 2001;7:927-32.

2 Anorlu RI, Fagbenro-Beyioku AF, Fagorala T, et al. Prevalence of Trichomonas vaginalis in patients with vaginal discharge in Lagos, Nigeria. Nigerian Postgrad Med J 2001;8:183-6.

3 Daly CC, Maggwa N, Mati JK, et al. Risk factors for gonorrhoea, syphilis, and trichomonas infections among women attending family planning clinics in Nairobi, Kenya. Genitourin Med 1994;70:155-61.

4 El Seoud SF, Abbas MM, Habib FS. Study of trichomoniasis among Egyptian male patients. J Egypt Soc Parasitol 1998;28:263-70.

5 Krieger JN. Trichomoniasis in men: old issues and new data. Sex Transm Dis 1995;22:83-96.

6 Krieger JN. Consider diagnosis and treatment of trichomoniasis in men [editorial; comment]. Sex Transm Dis 2000;27:241-2.

7 Pastorek JG 2nd, Cotch MF, Martin DH, et al. Clinical and microbiological correlates of vaginal trichomoniasis during pregnancy. Clin Infect Dis 1996;23:1075-80.

8 Morency P, Dubois MJ, Gresenguet G, et al. Aetiology of urethral discharge in Bangui, Central African Republic. Sex Transm Infect 2001;77:125-9.

9 Sorvillo F, Kovacs A, Kerndt P, et al. Risk factors for trichomoniasis among women with human immunodeficiency virus (HIV) infection at a public clinic in Los Angeles County, California: implications for HIV prevention. Am J Trop Med Hyg 1998;58:495-500.

10 Crosby R, DiClemente RJ, Wingood GM, et al. Predictors of infection with Trichomonas vaginalis: a prospective study of low income African-American adolescent females. Sex Transm Infect 2002;78:360-4.

11 Niccolai LM, Kopicko JJ, Kassie A, et al. Incidence and predictors of reinfection with Trichomonas vaginalis in HIV-infected women. Sex Transm Dis 2000;27:284-8.

12 Spinillo A, Bernuzzi AM, Cevini C, et al. The relationship of bacterial vaginosis, Candida and Trichomonas infection to symptomatic vagnitis in postmenopausal women attending a vaginitis clinic. Maturitas 1997;27:253-60

13 Mbizvo EM, Msuya SE, Stray-Pedersen B, et al. Determinants of repoductive tract infections among asymtpomatic women in Harare, Zimbabwe. Cent Afr J Med 2001;47:57-64.

14 Lo M, Reid M, Brokenshire M. Epidemiological features of women with trichomoniasis in Auckland sexual health clinics: 1998-99. NZ Med J 2002;115:U119.

15 Reynolds M, Wilson J. Is Trichomonas vaginalis still a marker for other sexually transmitted infections in women? Int J STD AIDS 1996;7:131-2.

16 Joyner JL, Douglas JM, Ragsdale S, et al. Comparative prevalence of infection with Trichomonas vaginalis among men attending a sexually transmitted diseases clinic. Sex Transm Dis 2000;27:236-40.

17 Wendel KA, Erbelding E, Gaydos CA, et al. Use of urine polymerase chain reaction to define the prevalence and clinical presentation of Trichomonas vaginalis infection in men attending an STD clinic. Sex Transm Infect 2003;79:151-3.

18 Pepin J, Sobela F, Deslandes S, et al. Etiology of urethral discharge in West Africa: the role of Mycoplasma genitalium and Trichomonas vaginalis. Bull World Health Organ 2001;79:118-26.

19 Watson-Jones D, Mugeye K, Mayaud P, et al. High prevalence of trichomoniasis in rural men in Mwanza, Tanzania: results from a population based study. Sex Transm Infect 2000;76:355-62.

20 Buve A, Weiss HA, Laga $M$, et al. The epidemiology of trichomoniasis in four African cities. AIDS 2001;15(Suppl 4):S89-96.

21 Moodley P, Wilkinson D, Connolly C, et al. Trichomonas vaginalis is associated with pelvic inflammatory disease in women infected with human immunodeficiency virus. Clin Infect Dis 2002;34:519-22.

22 Franklin TL, Monif GR. Trichomonas vaignalis and bacterial vaginosis. Coexistence in vaginal wet mount preparations from pregnant women. J Reprod Med 2000;45:131-4.

23 Schneider H, Coetzee N, Fehler HG, et al. Screening for sexually transmitted diseases in rural South African women. Sex Transm Infect 1998;74(Suppl 1):S147-52.

24 Ryan KA, Zekeng L, Roddy RE, et al. Prevalence and prediction of sexually transmitted diseases among sex workers in Cameroon. Int J STD AIDS 1998;9:403-7

25 Sayed el-Ahl SA, el-Wakil HS, Kamel NM, et al. A preliminary study on the relationship between Trichomonas vaginalis and cervical cancer in Egyptian women. J Egypt Soc Parasitol 2002;32:167-78.

26 Bowden FJ, Garnett GP. Trichomonas vaginalis epidemiology: parameterising and analysing a model of treatment interventions. Sex Transm Infect 2000;76:248-56.

27 Krieger JN, Jenny C, Verdon $M$, et al. Clinical manifestations of trichomoniasis in men. Ann Intern Med 1993;1 18:844-9.

28 Jackson DJ, Rakwar JP, Chohan B, et al. Urethral infection in a workplace population of East African men: evaluation of strategies for screening and management. J Infect Dis 1997; 175:833-8.

29 Hobbs MM, Kazembe P, Reed AW, et al. Trichomonas vaginalis as a cause of urethritis in Malawian men [see comments]. Sex Transm Dis 1999:26:381-7.

30 Krieger JN, Verdon $M$, Siegel N, et al. Natural history of urogenital trichomoniasis in men. J Urol 1993;149:1455-8. 
31 Kaydos SC, Hobbs MM, Price MA, et al. Sites of Trichomonas vaginalis infection in the genitourinary tract of Malawian men. Int J STD AIDS 2001; 12(Suppl 2):38.

32 Schwebke JR, Morgan SC, Pinson GB. Validity of self-obtained vaginal specimens for diagnosis of trichomoniasis. J Clin Microbiol 1997;35:1618-9

33 Wiesenfeld HC, Lowry DL, Heine RP, et al. Self-collection of vaginal swabs for the detection of Chlamydia, gonorrhea, and trichomoniasis: opportunity to encourage sexually transmitted disease testing among adolescents. Sex Transm Dis 2001;28:321-5.

34 Kingston MA, Bansal D, Carlin EM. "Shelf life" of Trichomonas vaginalis. Int J STD AIDS 2003:14:28-9.

35 Wendel KA, Erbelding EJ, Gaydos CA, et al. Trichomonas vaginalis polymerase chain reaction compared with standard diagnsotic and therapeutic protocols for detection and treatment of vaginal trichomoniasis. Clin Infect Dis 2002;35:576-80.

36 Ohlemeyer CL, Hornberger LL, Lynch DA, et al. Diagnosis of Trichomonas vaginalis in adolescent females: InPouch TV culture versus wet-mount microscopy. J Adolesc Health 1998;22:205-8.

37 Schwebke JR, Venglarik MF, Morgan SC. Delayed versus immediate bedside inoculation of culture media for diagnosis of vaginal trichomonosis. J Clin Microbiol 1999;37:2369-70.

38 Lara-Torre E, Pinkerton JS. Accuracy of detection of Trichomonas vaginalis organisms on a liquid-based papanicolaou smear. Am J Obstet Gynecol 2003:188:354-6.

39 Herzberg AJ, Silverman JF. Detection of Trichomonas vaginalis in endocervical and ectocervical smears. Diagn Cytopathol 1996;14:273-6.

40 Waghorn DJ, Tucker PJ, Chia Y, et al. Collaborative approach to improve the detection and management of trichomoniasis in a low prevalence district. Int J STD AIDS 1998;9:164-7.

41 Wiese W, Patel SR, Patel SC, et al. A meta-analysis of the Papanicolaou smear and wet mount for the diagnosis of vaginal trichomoniasis. Am J Med 2000;108:301-8.

42 Patel SR, Wiese W, Ohl CA, et al. Systematic review of diagnostic tests for vaginal trichomoniasis. Infect Dis Obstet Gynecol 2000;8:248-57.

43 DeMeo LR, Draper DL, McGregor JA, et al. Evaluation of a deoxyribonucleic acid probe for the detection of Trichomonas vaginalis in vaginal secretions. Am J Obstet Gynecol 1996;174:1339-42.

44 Kengne P, Veas F, Vidal N, et al. Trichomonas vaginalis: repeated DNA target for highly sensitive and specific polymerase chain reaction diagnosis. Cell Mol Biol (Noisy-Le-Grand) 1994;40:819-31.
45 Lawing LF, Hedges SR, Schwebke JR. Detection of trichomonosis in vaginal and urine specimens from women by culture and PCR. J Clin Microbiol 2000;38:3585-8.

46 Kaydos SC, Swygard H, Wise SL, et al. Development and validation of a PCR enzyme-linked immunosorbent assay with urine for use in clinical research settings to detect Trichomonas vaginalis in women. J Clin Microbiol 2002;40:89-95.

47 Kaydos-Daniels SC, Miller WC, Hoffman I, et al. Validation of a urine-based PCR-enzyme-linked immunosorbent assay for use in clinical research settings to detect Trichomonas vaginalis in men. J Clin Microbiol 2003:41:318-23.

48 Vishwanath S, Talwar V, Prasad R, et al. Syndromic management of vaginal discharge among women in a reproductive health clinic in India. Sex Transm Infect 2000;76:303-6.

49 Gulmezoglu AM Garner P. Trichomoniasis treatment in women: a systematic review. Trop Med Int Health 1998;3:553-8.

50 duBouchet L, McGregor JA, Ismail M, et al. A pilot study of metronidazole vaginal gel versus oral metronidazole for the treatment of Trichomonas vaginalis vaginitis. Sex Transm Dis 1998;25:176-9.

51 Schmid GP, Narcisi EM, Mosure D, et al. Prevalence on metronidazoleresistant Trichomonas vaginalis in a gynecology clinic. J Reprod Med 2001;46:545-9.

52 Narcisi EM, Secor WE. In vitro effect of tinidazole and furazolidone on metronidazole-resistant Trichomonas vaginalis. Antimicrob Agents Chemother 1996:40:1121-5.

53 Sobel JD, Nyirjesy P, Brown WJ. Tinidazole therapy for metronidazolereistant vaginal trichomoniasis. Clin Infect Dis 2001;33:1341-6.

54 Anonymous. Sexually transmitted diseases treatment guidelines. Centers for Disease Control and Prevention: MMWR 2002;51(RR-6):44-5.

55 Sutton MY, Sternberg M, Nsuami M, et al. Trichomoniasis in pregnant human immunodeficiency virus-infected and human immunodeficiency virusuninfected congolese women: prevalence, risk factors, and association with low birth weight. Am J Obstet Gynecol 1999:181:656-62.

56 Klebanoff MA, Carey JC, Hauth JC, et al. Failure of metronidazole to prevent preterm delivery among pregnant women with asymptomatic Trichomonas vaginalis infection. N Engl J Med 2001;345:487-93.

57 Czeizel AE, Rockenbaver M. A population based case-control teratologic study of oral metronidazole treatment during pregnancy. Br J Obstet Gynaecol 1998;105:322-7.

58 DuBouchet L, Spence MR, Rein MF, et al. Multicenter comparison of clotrimazole vaginal tablets, oral metronidazole, and vaginal suppositories containing sulfanilamide, aminacrine hydrochloride, and allantoin in the treatment of symptomatic trichomoniasis. Sex Transm Dis 1997;24:156-60. 